\title{
A novel application of ultrasonic Lamb waves: studying the effects of adhesive on the inspection of coating debonding in a three-layer waveguide
}

\author{
Mohammad Mehrabi ${ }^{\mathrm{a}}$, Mohammad Hossein Soorgee ${ }^{\mathrm{a}, *}$, Hossein Habibi $^{\mathrm{b}}$, Vassilios \\ Kappatos $^{\mathrm{c}}$ \\ ${ }^{a}$ Faculty of Mechanical and Energy Engineering, Shahid Beheshti University, Tehran, Iran. \\ ${ }^{b}$ School of Computing, Engineering, and Digital Technology, Teesside University, \\ Middlesbrough TS1 3BX, United Kingdom. \\ ${ }^{C}$ Hellenic Institute of Transport (HIT), Center for Research and Technology Hellas \\ (CERTH), 57001, Thermi, Thessaloniki, Greece. \\ *Corresponding author e-mail: mh_soorgee@sbu.ac.ir
}

\section{Abstract}

In this research, a thorough experimental and numerical study is conducted to investigate a novel application of the ultrasonic Lamb waves which is its capability to detect of the coating disbond in a double-layer waveguide (aluminium-adhesive) and a triple-layer waveguide (aluminium-adhesive-coating). The experimental tests are set up and implemented to evaluate how the attenuation of Lamb waves is affected by the adhesive bonding beside the contribution of the coating layer to the behaviour of Lamb waves. The semi-analytical finite element (SAFE) method is employed to analyse and identify the properties of guided wave modes propagating along the coated structures. In the presence of additive white Gaussian noise (AWGN), a range of important indices including energy index (EI), amplitude index (AI), pulse width index (PWI), and the time of flight index (ToFI) are worked out in association with the disbond length. It is inferred that a) even in the very noisy environment of $0 \mathrm{~dB}$ SNR, the Lamb waves are effectively able to identify debonding defects particularly using the EI, giving sensitivity rates $105 \%$ and $30 \%$ for triple-layer and double-layer, respectively; b) the contribution of adhesive to the absorption of the guided wave energy is $30 \%, 40 \%$, and $50 \%$ in EI, AI, and PWI, respectively, corroborating the value of the features to distinguish the debonding between each layer of bonded structures. 
Keywords: Ultrasonic Lamb waves; Adhesive Bond; Debonding inspection;

Viscoelastic Coating; Frequency Selection.

\section{Introduction}

Among numerous methods for protecting structures against environmental damages, applying the polymeric coatings is one of the most common efficient ways to prevent metal structures from corrosion and increase their life span [1]. Some viscoelastic coatings do not adhere to the substrate spontaneously and require an intermediate material to fill and bind properties with the material underneath. Adhesive bonding, as intermediates, is one of the most known and adopted joining method reducing extremely the cost and the weight of structures comparing with other joining methods, namely screwing and riveting. It is also noteworthy that the adhesives do not interfere with the internal structure of adherends [2].

Inadequate surface preparation, external loading, as well as physical aging are the common factors that could negatively affect the bonding quality, leading to the adhesive degradation with possible catastrophic failures of the structure [3]. Non-destructive evaluation (NDE) methods such as conventional ultrasonic tests including contact [4-11] and non-contact methods [12-14], X-ray radiography [15], and thermography [16] are widely used for bonding evaluation. However, the above-mentioned NDE methods have significant weaknesses like the requirement of access to the inspection point, high time cost, the limited ability to inspect long distances, and vulnerability to the influence of environment (e.g., temperature and moisture) [17].

Ultrasonic guided waves (UGWs) have been widely used to inspect elongated and complicated structures in order to detect, locate, characterize defects, and overcome mentioned deficiencies [18]. Recently, the assessment of the adhesively bonded structures based on guided ultrasonic Lamb waves has been further investigated due to its simplicity, cheapness, and high-bandwidth excitation [19]. Among various imperfections in such structures, delamination [20] and debonding [21,22] are more intricate, as they are invisible and inaccessible. On this basis, the effectiveness of the UGW in bondline inspection lies in choosing a fine-tuned mode and frequency, and in this regard, different techniques have been proposed [22-24]. Giurgiutiu et al. [25] employed electromechanical impedance in different bond lengths to exhibit the potential of piezoelectric wafer active sensors (PWAS) in adhesive bond monitoring; they found 
out the method is sensitive to this kind of defects. This conclusion was confirmed by Park [26] as well. As pointed out by Rokhlin $[5,27]$ both interface-wave and Lamb wave are useful to examine the adhesive bond quality based on both the phase delay and the phase velocity. In particular, numerous researchers have made use of specific Lamb waves modes, in which both asymmetric and symmetric modes were successfully able to monitor the integrity of three-layer bonding structures [28-31]. Using zero-group velocity Lamb wave modes generated by laser-ultrasound, Spytek et al. [32] identified and imaged debonding defects between various layers of epoxy-bonded aluminium plates. Similar to the previous research, Wojtczak et al. [33] proposed a laser-based guided wave technique to detect and visualize disbond at the interface of steel-concrete beams in adhesive joints. The effectiveness of the method, however, was quite dependent on the selected frequency as well as the location of excitation.

Taking advantage of UGW interaction with disbond defect, some features can be extracted to characterize the bonding weakness [34-36], among which time-based features have been more common and are the only prominent mechanism for this purpose [37-41]. Though Van Velsor [42] identified a feature based on the frequency content to study coating debonding in a multi-layered hollow cylinder using shear horizontal ( $\mathrm{SH}$ ) guided waves. Moreover, Bostron [43] conducted a successful bond inspection at the boundary between a viscoelastic coating and a steel plate by the guided interface waves, based on both frequency-based and time-based features. Some other numerical and analytical studies have been also proposed $[44,45]$, but overall more effective works still entail for the understanding of the interaction of guided waves with degraded bonding.

This paper presents a complete and detailed numerical-experimental investigation of applying Lamb waves in the evaluation of disbond defects at the adherend-adhesive interface. The novelty of the research is associated with identifying the contribution of adhesive to the absorption of the guided wave energy while bonded to a viscoelastic layer, and subsequently its effect on diagnosing the disbond defect in noisy environments. The method helps to discern the debonding between each layer of bonded joints. To this end, an exhaustive set of experiments was carried out on two cases: a two-layer waveguide (metal plate-adhesive) and a three-layer waveguide (metal plate-adhesive-coating). In the former, the disbond between a metal plate and a thin adhesive layer that is one fifth the thickness of the metal plate was investigated. Although the adhesive material causes the Lamb waves to be damped, the contribution of adhesive along with coating layer in the 
wave attenuation is not accurately identified. Therefore, the latter case is designed to determine the role of each adhesive and coating in the wave attenuation. In this vein, by adding a coating on adhesive layer, the debonding between the metal plate and protective coating (coating and adhesive aggregately) is examined. In fact, the wave propagation in the protective coating is investigated in the same way as for the adhesive layer to let us study how the adhesive is contributed to the guided wave propagation and its impact on the disbond inspection. A schematic diagram demonstrating both cases are shown in Fig. 1.
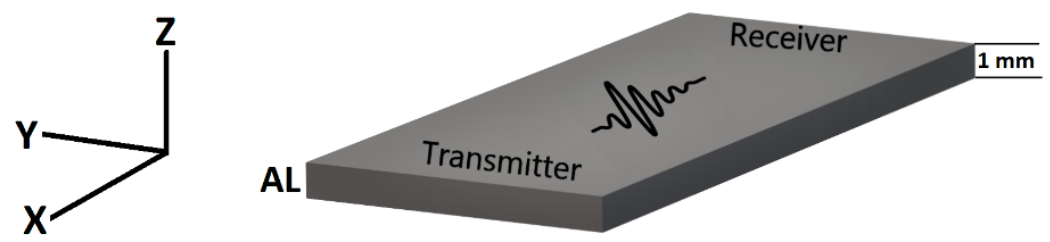

(a)

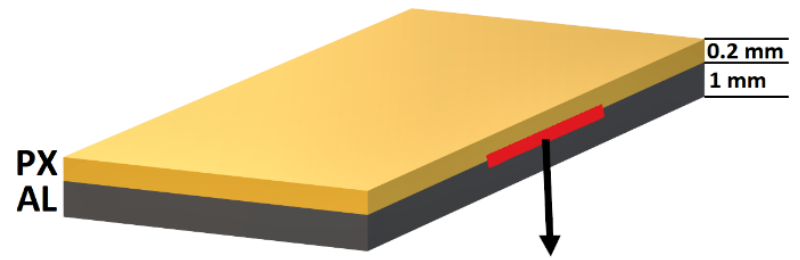

(b)

Debonding area

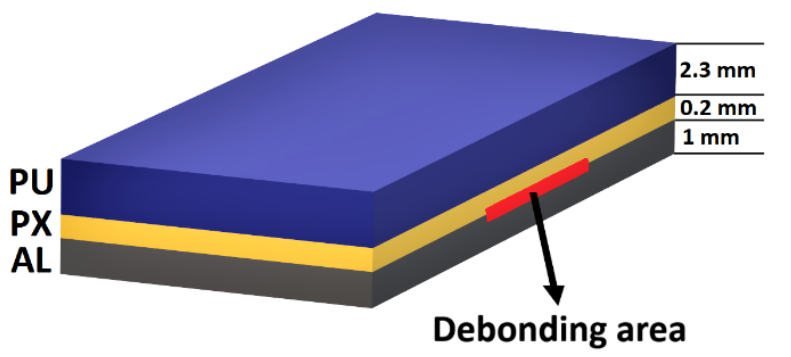

(c)

Fig. 1. Schematic diagram of the experimental setup (drawing not to scale): (a) aluminium bare plate (Al), (b) debonding in two-layer waveguide between $\mathrm{Al}$ and $\mathrm{PX}$ adhesive bond, and (c) debonding in three-layer waveguide between $\mathrm{Al}$ and the protective coating (PX-PU).

The paper is organized as follows. A critical review of earlier studies on the bond evaluation was presented in Section 1. A theoretical investigation of the guided waves in multilayer structures is discussed in Section 2. The details of the experimental setup are provided in Section 3. The theoretical results are found in Section 4.1 which is followed by the assessment of extracted signal features at both free-noise and noisy environments in Section 4.2 and Section 4.3, respectively. Lastly, it ends up by the conclusions in Section 5 . 


\section{Guided Waves in Multi-layer Structures}

Multi-layer engineering structures are extensively used in various industrial applications such as pipelines with viscoelastic coatings and composite materials in aviation [46]. The study of guided waves in these media requires a deep understanding of guided wave attributes like dispersion curves, wave structure and mode conversion [18]. A crucial step in the inspection of a multi-layer structures and components using guided waves is the dispersion analysis which represents the dependence of phase speed and attenuation on frequency. Researchers have widely used mathematical solutions to solve these problems [47-49]. The solution of the dispersion can be derived analytically by the matrix method [50]. Both the Transfer Matrix Method (TMM) and the Global Matrix Method (GMM) are capable of solving dispersion problems [51]. However, the TMM is unstable at high frequencies relative to layer thickness. On the one hand GMM is not as sensitive as TMM to the high frequencies, but on the other hand as the number of layers increases the computational cost will be significantly increased [52]. Unlike the analytical models that are unable to solve complex cross-sectional geometries, the semi-analytical finite element method (SAFE) overcomes these deficiencies [53]. In the SAFE method, the structure is discretized into two parts: first, the cross-section that uses finite elements method (FEM) and second, the wave propagation direction taking advantage of the analytical solution. Recently, several scholars benefited from this technique to find a proper solution to guided wave problems in viscoelastic media $[54,55]$.

Thanks to the versatile dispersion calculator software, GUIGUW, one can easily employ the SAFE method for extracting the dispersion curves of complex multi-layer structures coated with viscoelastic layers [56]. Taking advantage of GUIGUW in this study, the SAFE method has been used to analyse the propagation of guided waves through two-layer and three-layer waveguides. The same as the configuration used in the experimental test, the two-layer sample includes an aluminium plate ( $\mathrm{Al}$ ) as the host substrate and Polymax (PX) adhesive as the thin layer of adhesive, while the polyurethane (PU) as the coating layer is bonded over the PX adhesive in the three-layer model (see Fig. 1). As the main assumption, the aluminium plate undergoes infinite lateral extension where the propagation of guided wave only in longitudinal direction of the structure is considered. Therefore, a 1-D SAFE model complies with conditions in the present work.

The 1-D SAFE model is discretized into the analytical solution and the FE model of cross-section at Y-Z plane. The former is used to solve eigenvalue problem and find the 
wavenumbers for a given frequency value along the direction of Lamb wave propagation (X axis). Note that selecting the number of elements in the waveguide layers considers a trade-off for accuracy and computational efficiency. The optimal number of elements, absorption coefficients, and material properties of each layer related to the SAFE model are given in Table 1.

Table 1. Material properties used in the SAFE model and experimental test. Notice that the attenuation coefficients of viscoelastic material $\left(\alpha_{L}\right.$ and $\left.\alpha_{T}\right)$ are used as inputs only for PU Coating.

\begin{tabular}{|c|c|c|c|c|c|c|c|c|c|}
\hline & \multicolumn{2}{|c|}{ Geometry } & \multicolumn{6}{c|}{ SAFE Inputs } & \\
\cline { 2 - 9 } Material & $\begin{array}{c}\text { Length } \\
(\mathrm{m})\end{array}$ & $\begin{array}{c}\text { Width } \\
(\mathrm{m})\end{array}$ & $\begin{array}{c}\text { Thickness } \\
(\mathrm{mm})\end{array}$ & $\begin{array}{c}\text { Elastic } \\
\text { Modulus } \\
(\mathrm{GPa})\end{array}$ & $\begin{array}{c}\text { Poisson's } \\
\text { Ratio }\end{array}$ & $\begin{array}{c}\text { Density } \\
\left(\mathrm{kg} / \mathrm{m}^{3}\right)\end{array}$ & $\begin{array}{c}\boldsymbol{\alpha}_{\mathrm{L}} \\
(\mathrm{Np} / \mathrm{mm})\end{array}$ & $\begin{array}{c}\boldsymbol{\alpha}_{\mathrm{T}} \\
(\mathrm{Np} / \mathrm{mm})\end{array}$ & $\begin{array}{c}\# \\
\text { Elem }\end{array}$ \\
\hline $\begin{array}{c}\text { Aluminium } \\
(3105)\end{array}$ & 1 & 0.8 & $1( \pm 1 \%)$ & 69 & 0.33 & 2700 & - & - & 5 \\
\hline $\begin{array}{c}\text { Polymax } \\
\text { Adhesive }\end{array}$ & 0.6 & 0.07 & $0.2( \pm 5 \%)$ & $5 \mathrm{e}-4$ & 0.45 & $1040( \pm 2 \%)$ & - & - & 8 \\
\hline $\begin{array}{c}\text { Polyurethane } \\
\text { Coating }\end{array}$ & 0.6 & 0.07 & $2.3( \pm 1 \%)$ & 0.1 & 0.38 & $1200( \pm 2 \%)$ & $\begin{array}{l}0.0036 \\
( \pm 3 \%)\end{array}$ & $\begin{array}{c}0.008 \\
( \pm 5 \%)\end{array}$ & 23 \\
\hline
\end{tabular}



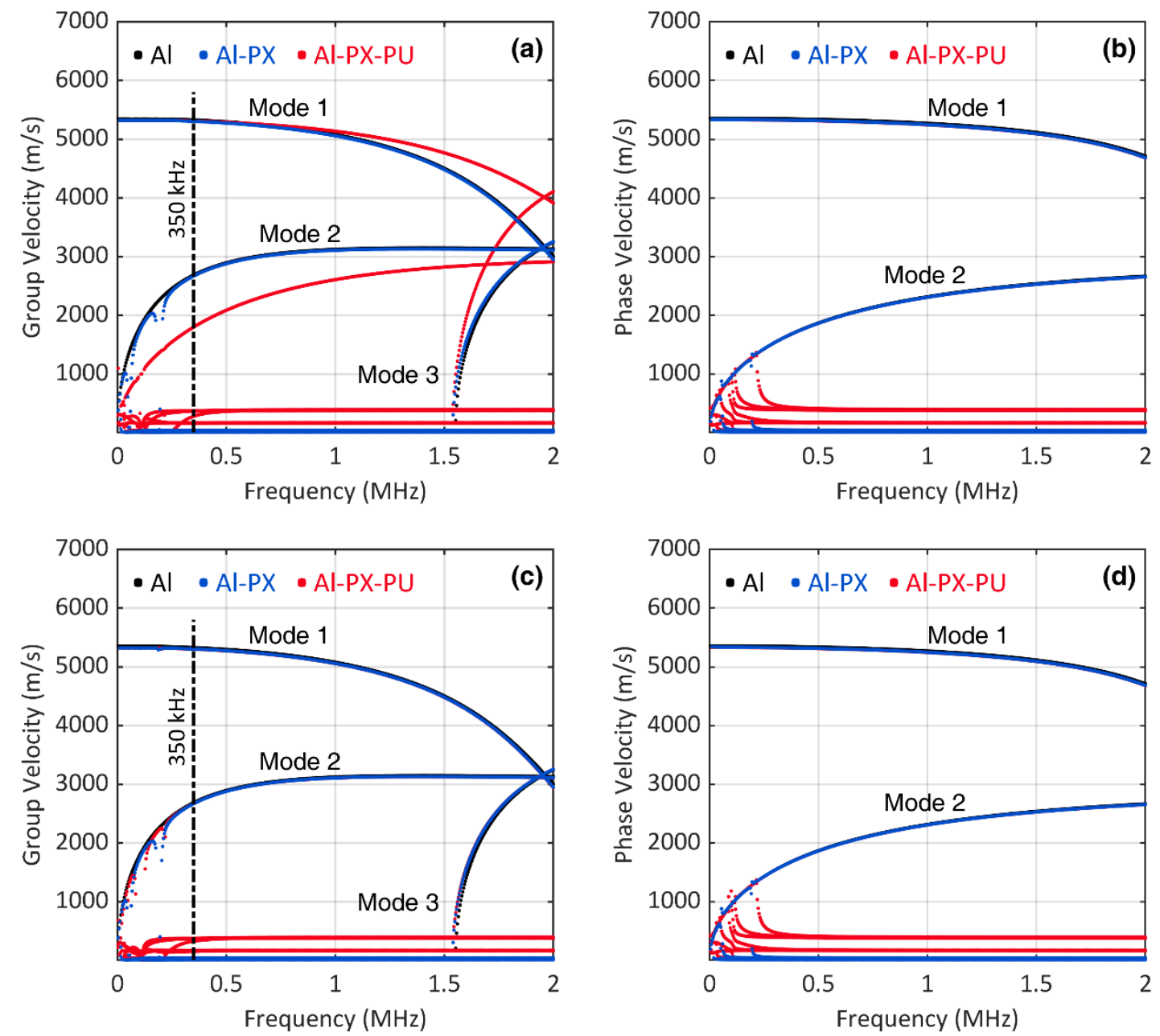

Fig. 2. Dispersion curves of group and phase velocities of Lamb waves obtained by the SAFE method in: aluminium bare plate Al (black dotted), two-layer Al-PX (blue dotted), and three-layer Al-PX-PU (red dotted). Notice that the PU in (a) and (b) considered viscoelastic and in (c) and (d) considered elastic.

The Dynamic Mechanical Analysis (DMA) is an effective tool for evaluating the viscoelastic behaviour of polymers based on the phase difference between strain and stress [57]. According to the DMA of PU and PX materials, the PU function is more similar to viscoelastic materials; consequently, only the PU is considered as a viscoelastic material in the SAFE model. Meanwhile, longitudinal and shear attenuations of PU are required as viscoelastic parameters in the SAFE model. The attenuation characteristics of viscoelastic materials can be derived from a comparison between the amplitude of longitudinal or shear waves propagated through the material of two different thicknesses [52]. Accordingly, the attenuation coefficients of PU are obtained, which is provided in Table 1. Nevertheless, the PU is examined as both elastic and viscoelastic to let us track the viscoelastic effect on the SAFE results and decrease the possible computational cost. 
The dispersion curves of group and phase velocities of Lamb waves using SAFE method presented in Fig. 2 and indicate that at the frequencies below $2 \mathrm{MHz}$, the presence of viscoelastic material (PU coating) does not have a noticeable impact on phase velocity, in contrast to the group velocity. Although the group velocity at the frequency range of the test (150 to $600 \mathrm{kHz}$ ) for both the viscoelastic and elastic models varies in the second mode, there is no significant difference at the mode 1, which is used as the inspection mode in this study. Fig. 2 also reveals that mode 1 and mode 2 related to the two-layer and three-layer waveguides are similar to the symmetric and asymmetric Lamb wave modes in the one-layer waveguide (Al). However, in a non-symmetric structure like those used in this study the symmetric Lamb mode might not be an appropriate name, as the mode shape does not present symmetry considering both surfaces of the structures. The numbers have thus been used for each mode. It is noteworthy that mode 3 appears above the velocity limit $(7000 \mathrm{~m} / \mathrm{s})$ assumed in in Fig. 2 (b) and (d).

\section{Experimental Setup and Procedures}

The final specimen used for the experiments is made of three different components: an aluminium substrate bonded with a PX adhesive layer to a PU coating over a finite size of its surface. The dimensions and the properties of materials are tabulated in Table 1. The PX adhesive is employed to apply the polyurethane coating to the aluminium plate surface, which is semi-liquid and requires at least five hours to cure at room temperature. Before applying PX, the surface of the plate should be thoroughly cleaned to make a perfect adhesive bond on the aluminium plate. A $0.2 \mathrm{~mm}$ thick paper tape is utilized to make a mould for the uniformity of adhesive bond. Thanks to the mould, the approximate $0.2 \mathrm{~mm}$ thickness of the adhesive is guaranteed, which is also visually inspected (see Fig. 3(a)). After curing, the paper tape is removed and only the PX adhesive bond remains. This is the procedure to fabricate the two-layer waveguide (Al-PX) shown in Fig. 3(b). To fabricate the three-layer waveguide (Al-PX-PU), all steps are the same as mentioned before, except that the PU coating must be bonded to the adhesive bondline immediately after applying the PX adhesive (see Fig. 3(c)). In order to minimize the error in results, the experiments were performed under the same circumstances (e.g. temperature and humidity) for both Al-PX and Al-PX-PU cases. The disbonds in the two-layer and threelayer waveguides are assumed to be at the Al-PX and Al-PXPU interfaces, respectively. A PX strip and a PXPU strip are eliminated from the plate to model the debonding defect in Al-PX and Al-PX-PU waveguides, respectively (see Fig. 1 and Fig. 3(d)). The lengths 
of the disbond region in both cases are 5, 10, 15, and $20 \mathrm{~cm}$. A general view of the experimental setup and the disbond region is shown in Fig. 3(d).

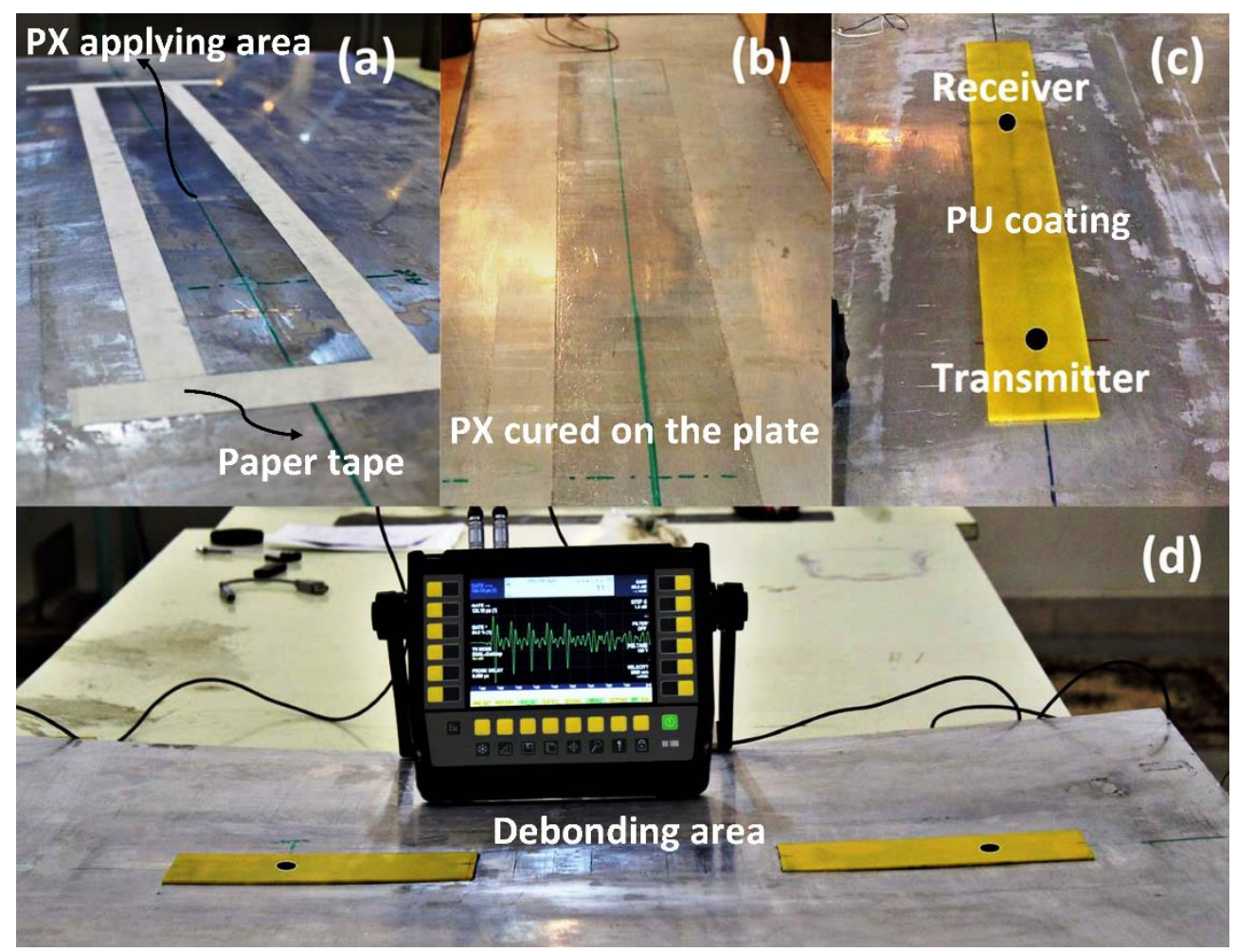

Fig. 3 Experimental setup: (a) the $0.2 \mathrm{~mm}$ paper tape to control the size and thickness of the adhesive, (b) the two-layer sample (Al-PX), (c) the three-layer sample (Al-PX-PU), and (d) the disbond region in three-layer sample alongside the ultrasonic flaw detector. Note that the piezo discs are bonded beneath the aluminium plate and their locations are demonstrated by black circles on PU.

In the present work, Lamb waves are generated and detected using a SM411 STEMINC piezoelectric ceramic disc of $8 \mathrm{~mm}$ diameter and a thickness of $0.5 \mathrm{~mm}$ with $300 \mathrm{kHz}$ resonant frequency that are coupled on the aluminium plate using adhesive. It should be noted that the mode that is mainly generated by the piezoelectric elements within the aluminium plate in the present study is the zero-order symmetric $\left(\mathrm{S}_{0}\right)$ Lamb mode. However, as mentioned in section 2 the wave mode that propagates through the two-layer and three-layer structures is no longer $S_{0}$ Lamb wave mode. Therefore, the mode 1 , analogous to the $\mathrm{S}_{0}$ Lamb mode in the aluminium plate is employed in both twolayer and three-layer samples as the inspection mode. The distance between the transmitter and the receiver is $45 \mathrm{~cm}$ and they are in the pitch-catch configuration. It is mentionable that the transmitter and receiver are bonded firmly beneath the plate by adhesive, and their locations are marked by the black circles on the PU coating (see Fig. 3(c)). As the input signal, a five-cycle square burst signal with centre frequency of 350 $\mathrm{kHz}$ is utilized, and the duration of signal measurement is $60 \mu \mathrm{s}$. The reasons for choosing 
the frequency of $350 \mathrm{kHz}$ are further discussed in section 4.1. As it is evident in Fig. 3(d), DIO-1000 STARMANS has been used as an ultrasonic diagnostic device to send and receive electric signals.

\section{Results and Discussion}

\subsection{Theoretical Results}

\subsubsection{Frequency Selection}

Although the piezoelectric operating frequency at this setup is about $300 \mathrm{kHz}$, they are not limited to their resonant frequencies and are capable of generating a frequency range [19]. What follows now are the considerations for checking which frequency is the most appropriate one for the disbond inspection in this research. Firstly, it is necessary to analyse the dispersion curves to find an appropriate mode and frequency. The phase velocity and group velocity dispersion curves of the aluminium bare plate, the two-layer waveguide (Al-PX), and the three-layer waveguide (Al-PX-PU) have been provided by SAFE technique in Fig. 2. Given that the mode 1 is considered as the inspection mode, the assessment of other modes is neglected. Also, the effect of the viscoelastic coating on mode 1 at frequencies below $1 \mathrm{MHz}$ is not evident in both the group and phase velocities.

Besides, in coated structures, the presence of the coating can severely reduce the received signal amplitude; this is more obvious in structures coated with viscoelastic materials like pipelines. Consequently, the excitability must be checked at the frequency range of the tests, which is the key to long-range inspections. In order to obtain the maximum penetration power in the coated waveguides, the amplitude (energy) of the signals at different frequencies must be compared. To this intent, the transmitter piezo is excited from $150 \mathrm{kHz}$ to $600 \mathrm{kHz}$, with a step frequency of $50 \mathrm{kHz}$. Then the amplitudes of the received signals are compared. A $2^{\text {nd }}$ order Butterworth filter has been employed to remove the signal noise. According to the results presented in Fig. 4(a), the frequency of $350 \mathrm{kHz}$ implies the highest associated energy and guarantees the excitability in all the three kinds of plate: Al, Al-PX, and Al-PX-PU. It should be mentioned that the type of transducer and its location on the waveguide can significantly alter the excitation power of Lamb wave modes. 
Last but not least, the sensitivity of mode 1 at $350 \mathrm{kHz}$ to the presence of the coating should be theoretically studied by the wave structure to determine if the selected mode and frequency will interact with debonding defect. Apart from dispersion curves, the displacement wave structure associated with each frequency can be simply plotted by GUIGUW software. The software makes use of eigenvector that corresponds to the eigenvalue problem of the wavenumber in a given frequency. Detail of which can be found in reference [56]. Fig. 4(b) indicates in-plane and out of plane displacement wave structure of three-layer waveguide at $350 \mathrm{kHz}$ for the Lamb wave mode 1 . The mode shapes are normalized with respect to the maximum in-plane displacement $\left(u_{x}\right)$. Generally, for bondline evaluation, it is advised to choose a mode of high-magnitude inplane displacement at the interface of the host structure (Al) and the protective coating layers (PX-PU) to provide sufficient sensitivity to the debonding defect [22]. It is readily apparent in Fig. 4(b) that the Lamb wave mode 1 undergoes the maximum in-plane displacement $\left(\mathrm{u}_{\mathrm{x}}\right)$ at the interface of $\mathrm{Al}$ and PX-PU which indicated by a black arrow, confirming the sensitivity of selected mode and frequency to the bonding condition. As a result, the Lamb wave mode 1 at the frequency of $350 \mathrm{kHz}$ has the adequate penetration capability in viscoelastic materials and is powerful enough to evaluate disbond defects.
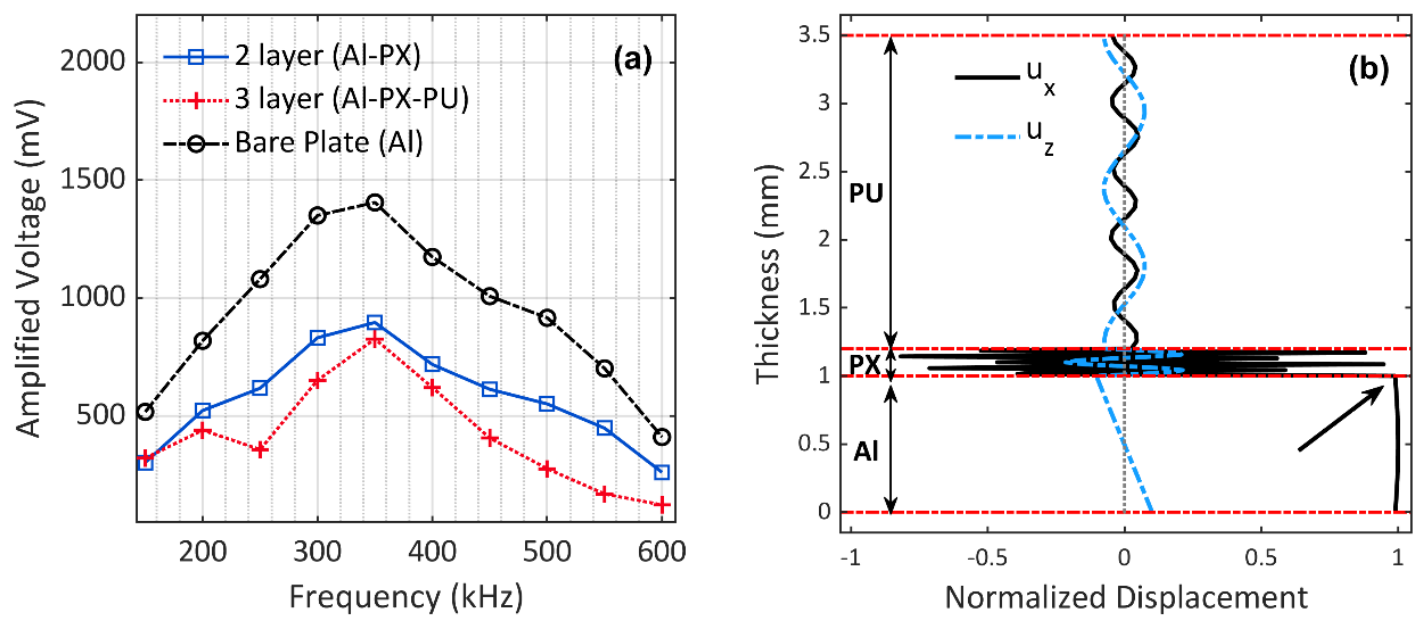

Fig. 4. (a) Amplitude excitability in different frequencies for all three waveguides. (b) In-plane (black solid) and out of plane (blue dashed) displacement wave structure at $350 \mathrm{kHz}$ for a Lamb wave mode 1 at the interface of the aluminium plate ( $\mathrm{Al})$ and the protective coating layers (PX-PU), normalized with respect to the maximum in-plane displacement $\left(u_{x}\right)$ that marked by a black arrow.

\subsubsection{Lamb Waves Interaction with Disbond}

The existence of a disbond region causes a change in the acoustic impedance and boundaries of a waveguide, which alters the characterizations of the guided Lamb waves 
namely amplitude, phase, and mode. The dispersion curves in Fig. 2 show that only the mode 1 and mode 2 can exist at $350 \mathrm{kHz}$ frequency. Therefore, mode conversion from the mode 1 to the mode 2 can take place when Lamb waves travel through the structural change (debonding area), which can change both wave velocity and waveform shape. Nevertheless, the comparison between the waveform of bare and coated plates in Fig. 5(a) shows that the waveform with the absence of adhesive or coating layers has undergone a little change, which means the presence of disbond defect slightly changes the excitation waveform, as shown in Fig. 5(b). Accordingly, the mode conversion can be overlooked in mode 1 .

Moreover, according to Fig. 2, one may consider mode 1 at $350 \mathrm{kHz}$ within the AlPX and Al-PX-PU samples as a non-dispersive mode. However, the experimental signals received in the defectless area in Fig. 5(a) reveal that the number of tone burst cycles has increased from 5 to 8, which can imply the dispersion phenomenon. Having these in mind, the following section attempts to extract some features based on the amplitude and phase factors to demonstrate the ability of the guided Lamb waves for evaluating the debonding defects between the waveguide layers, both in noise-free and very noisy environments.
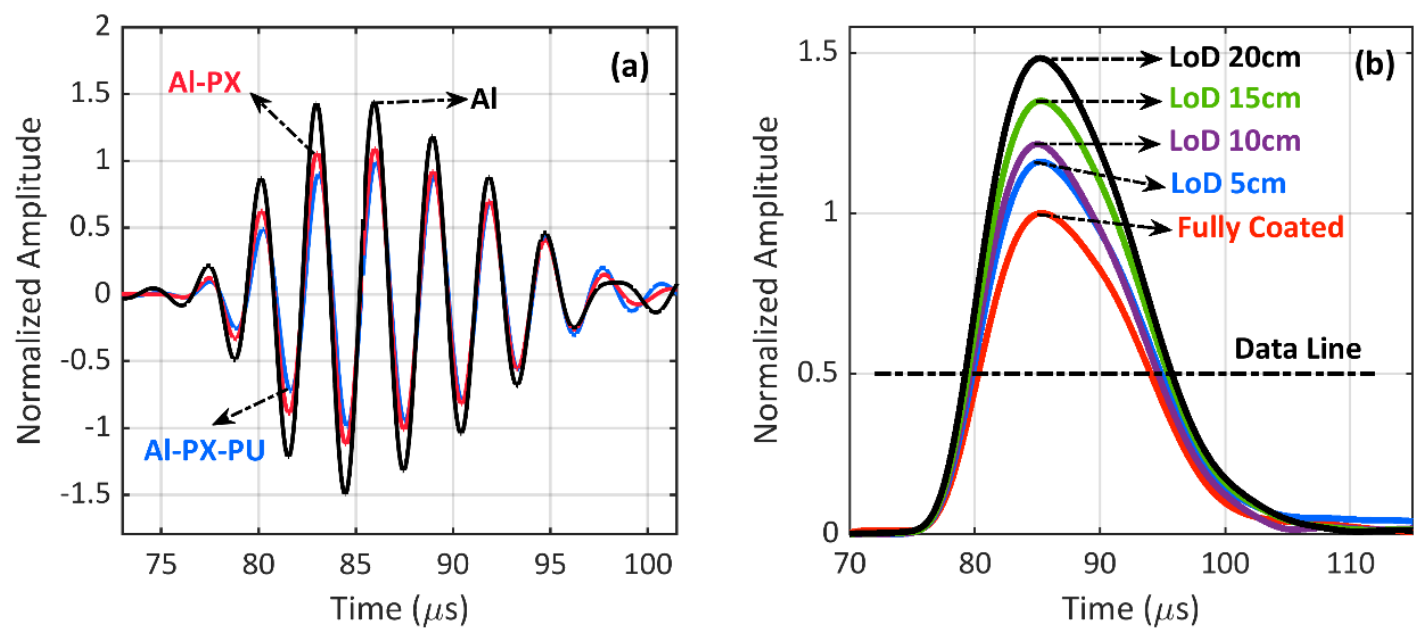

Fig. 5. (a) Experimental signal amplitudes normalized with respect to the three-layer sample related to the bare plate (Al), two-layer (Al-PX), and three- layer (Al-PX-PU). (b) Normalized amplitude envelope with respect to the fully-coated region at different lengths of debonding $(\mathrm{LOD})$ in the three-layer sample.

\subsection{Signal Indices Results}

Generally, features are obtained by the difference between the pristine and defected signals (see Fig. 5(b)). Four indices have been extracted from signals, including peak to peak amplitude, signal energy, signal width, and time of flight. All features are based on 
percentages to be comparable. It is notable that only the first wave packet of each signal is taken into account to extract the indices.

\subsubsection{Amplitude Index (AI)}

The first and most conventional way to determine an interfacial weakness is the amplitude index (AI) which is calculated by the maximum peak to peak amplitude of waveform. As the Lamb wave travels through a multi-layer waveguide, it loses energy and leaks into adjacent attenuative layers. The wave then undergoes trivial attenuation in the region without coating. Note that the attenuation of Lamb waves in viscoelastic materials depends on various factors, such as material damping, leakage, test frequency, geometric spreading, and wave dispersion [58]. One may expect that the amplitude must be decreased by the dispersion phenomenon, but when the disbond length increases, the impact of amplitude reduction is neglectable in comparison with amplitude increase in the absence of the coating. Therefore, this study focuses only on the first two factors. The amplitude index is calculated by:

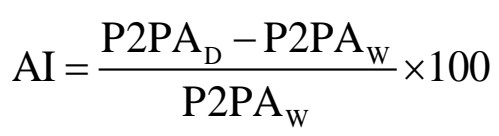

In the above equation, $\mathrm{AI}$ is the amplitude index, $\mathrm{P} 2 \mathrm{PA} \mathrm{A}_{\mathrm{D}}$ is the peak to peak amplitude of the signal related to the disbonded case, and P2PAw is the peak to peak amplitude of the well-bonded case. As depicted in Fig. 6 the presence of both the PX adhesive and double layer PX-PU on aluminium plate causes the energy of the Lamb waves to be damped. In other words, as disbond length increases from $5 \mathrm{~cm}$ to $20 \mathrm{~cm}$, the waveform amplitude increases as well in both cases. 


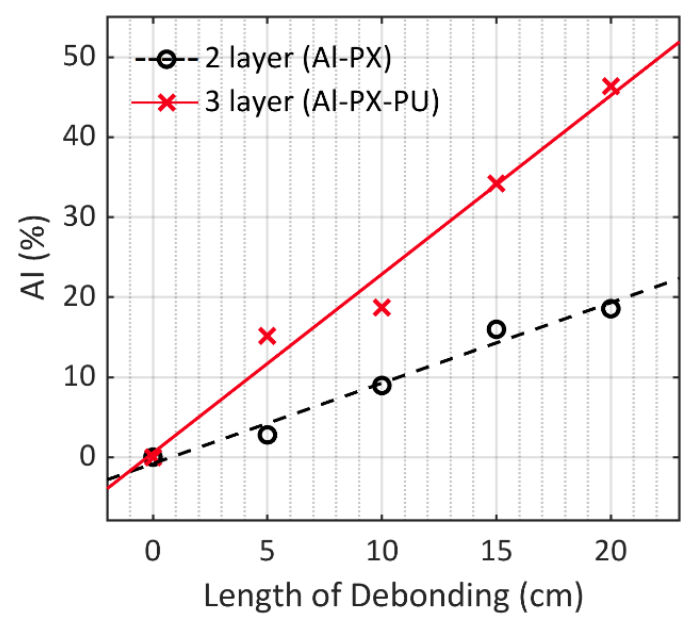

Fig. 6. Amplitude Index (AI) vs. length of debonding in both Al- PX and Al-PX-PU.

\subsubsection{Energy Index (EI)}

The energy index (EI) is calculated based on the signal energy value representing the area below the squared amplitude of the waveform. This has been done by squaring the normalized filtered signals followed by trapezoidal integration rule in MATLAB environment. Equation. 2 shows the formula used for EI calculation.

$$
E I=\frac{\int\left(S_{D}\right)^{2}-\int\left(S_{W}\right)^{2}}{\int\left(S_{W}\right)^{2}} \times 100
$$

In the above equation, EI is the energy index, $\mathrm{S}_{\mathrm{D}}$ is the signal corresponding to the disbond case, and $\mathrm{S}_{\mathrm{W}}$ is the wave packet signal related to the well-bonded case. It might be deemed that the EI is quite similar to the AI, but this is not the case. This can be nuanced by the fact that the duration of the wave packet in a dispersive media increases as the disbond length increases, resulting in an increase in the area of the amplitude envelope.

In both cases of Al-PX-PU and Al-PX, the fully coated plate has the smallest area under the wave packet and carries the lowest energy. Therefore, it is expected that the waveform that travels along the disbond zone bears more energy than the well-bonded zone as illustrated in Fig. 7. For all the tests, the range of the area is from $74 \mu$ s to $110 \mu \mathrm{s}$ and in both cases of Al-PX and Al-PX-PU, the feature trend is very similar to the previous index shown in Fig. 6. 


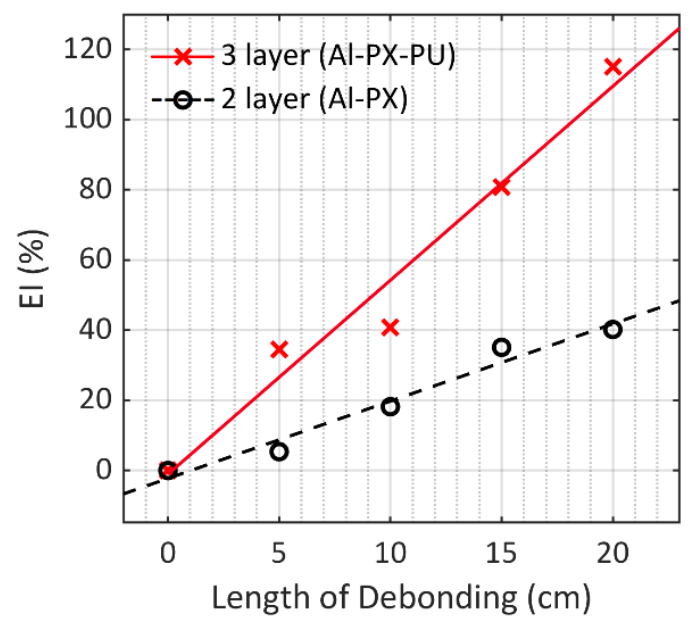

Fig. 7. Energy Index (EI) vs. length of debonding in both Al-PX and Al-PX-PU.

\subsubsection{Pulse Width Index (PWI)}

Another interesting feature is the pulse width index (PWI) which involves wave packet geometry. As its name implies, this feature is obtained by the width of the amplitude envelope in a specified amplitude ratio at $6 \mathrm{~dB}$ drop from the maximum amplitude of fully-coated sample, marked as "data line" in Fig. 5(b). The start and end points of the time range are determined by the data line depicted in Fig. 5(b). The height of the data line is half of the fully-coated amplitude. The index is calculated by the following equation:

$$
\mathrm{PWI}=\frac{\mathrm{W}_{\mathrm{D}}-\mathrm{W}_{\mathrm{W}}}{\mathrm{W}_{\mathrm{W}}} \times 100
$$

PWI is the pulse width index, $\mathrm{W}_{\mathrm{D}}$ is the width of amplitude envelope related to the disbond region signal in the data line, and $\mathrm{W}_{\mathrm{W}}$ is the envelope width of the well-bonded region in the same data line (see Fig. 5(b)). As expected, as the disbond length increases the amplitude envelope widens, which can be seen in Fig. 8. The feature trend is similar to the two previous features, but with a slight alternation. 


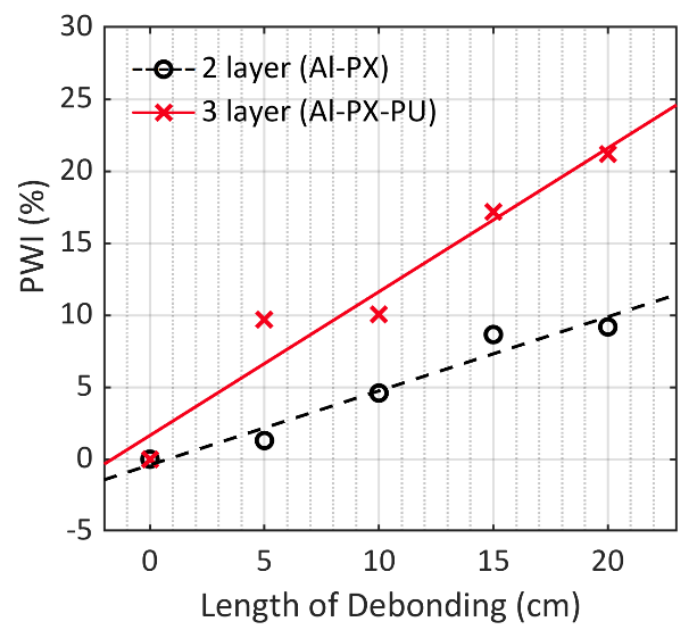

Fig. 8. Pulse Width Index (PWI) vs. length of debonding in both Al-PX and Al-PX-PU.

\subsubsection{Time of Flight Index (ToFI)}

The time of flight $(\mathrm{ToF})$ represents the arrival time of related wave packet to the receiver. As Lamb waves travel through an attenuative medium, the waves not only lose their energy but also decrease their velocity, contrary to bulk waves, which only lose their amplitude in attenuative media. This feature has great potential to become an attractive feature of the guided wave to assess debonding defects in multi-layer structures. Since the distance between transmitter and receiver is constant in all cases of the current research, it is concluded that if the length of debonding increases, the arrival time of the guided waves drops slightly. Meanwhile, it must be noted that based on dispersion curves indicated in Fig. 2(a), the difference between group velocities of mode 1 in Al, Al-PX and Al-PX-PU cases is not considerable, which goes the same for experimental results. Fig. 5(b) reveals that the arrival times of signals related to the defected samples are pretty close together. However, this little shift of phase can be due to the presence of absorbing materials (adhesive and coating). The theoretical and experimental group velocities of three above-mentioned cases in the fully-coated region, at $350 \mathrm{kHz}$ frequency are provided in Table 2. According to the table, the maximum difference between theoretical group velocity and experiments is about $1 \%$, confirming the accuracy of the SAFE model.

Table 2. Theoretical (based on the SAFE technique) and experimental group velocities of Al, Al-PX, and Al-PX-PU samples in defectless area.

\begin{tabular}{llll}
\hline Sample & Al & Al-PX & AI-PX-PU \\
\end{tabular}




\begin{tabular}{lccc}
\hline Theoretical $\mathrm{Cg}(\mathrm{m} / \mathrm{s})$ & 5327 & 5313 & 5311 \\
\hline Experimental $\mathrm{Cg}(\mathrm{m} / \mathbf{s})$ & 5330 & 5328 & 5326 \\
\hline
\end{tabular}

The theoretical travel time of mode 1 from the actuator to the receiver, for Al-PX and Al-PX-PU cases are represented as Equation. 4 and Equation. 5, respectively.

$$
\begin{gathered}
\mathrm{ToF}_{\mathrm{Al}-\mathrm{PX}}=\frac{\mathrm{LoD}}{\mathrm{C}_{\mathrm{Al}}}+\frac{\mathrm{L}-\mathrm{LoD}}{\mathrm{C}_{\mathrm{Al}-\mathrm{PX}}}, \\
\mathrm{ToF}_{\mathrm{Al}-\mathrm{PX}-\mathrm{PU}}=\frac{\mathrm{LoD}}{\mathrm{C}_{\mathrm{Al}}}+\frac{\mathrm{L}-\mathrm{LoD}}{\mathrm{C}_{\mathrm{Al}-\mathrm{PX}-\mathrm{PU}}}
\end{gathered}
$$

In the above equations, $\mathrm{C}_{\mathrm{Al}}, \mathrm{C}_{\mathrm{Al}-\mathrm{PX}}$, and $\mathrm{C}_{\mathrm{Al}-\mathrm{PX}-\mathrm{PU}}$ are the group velocities correspond to the mode 1 in the aluminium bare plate, two-layer Al-PX, and three-layer Al-PX-PU, respectively. The $\mathrm{L}$ is the overall distance between the transducers and $\mathrm{LoD}$ is the length of disbond region.

Another index that should be calculated and discussed is ToFI, which is the relative difference between ToF of the Lamb wave mode 1 in well-bonded case and damaged case, determined by

$$
\mathrm{ToFI}=\frac{\mathrm{ToF}_{\mathrm{w}}-\mathrm{ToF}_{\mathrm{D}}}{\mathrm{ToF}_{\mathrm{w}}} \times 100,
$$

where ToFI is the ToF index, $\mathrm{ToF}_{\mathrm{W}}$ and $\mathrm{ToF}_{\mathrm{D}}$ are the ToFs in the well-bonded case and the debonding case, respectively.

As it is concluded from the data provided in Table 2, group velocities in these cases are very close to each other which causes a small difference in ToFs and leads to a small amount of ToFI. Based on this phenomenon, the ToF index is theoretically examined in both the Al-PX and Al-PX-PU cases. Fig. 9(a) presents the ToFI that corresponds to theoretical (SAFE) model only. As shown in Fig. 9, there is less than $0.15 \%$ of ToFI for the largest debonded case, which makes it almost impossible to verify the data by experiments. 
Similar to the results obtained by the SAFE method, these results have been also extracted from experimental tests. TOFI for experimental tests is depicted in Fig. 9(b). It should be noted that the arrival time of each wave packets has been considered as the time of the maximum point at amplitude envelope shown in Fig. 5(b). As it is expected, due to uncertainties in experimental tests, ToFI has a fluctuating trend in Fig. 9(b). The reason for such discrepancies between theoretical and experimental results may be that the group velocities of studied cases are close to each other at mode 1. Consequently, in the present work, ToFI does not seem to be a reliable index for debonding evaluation.
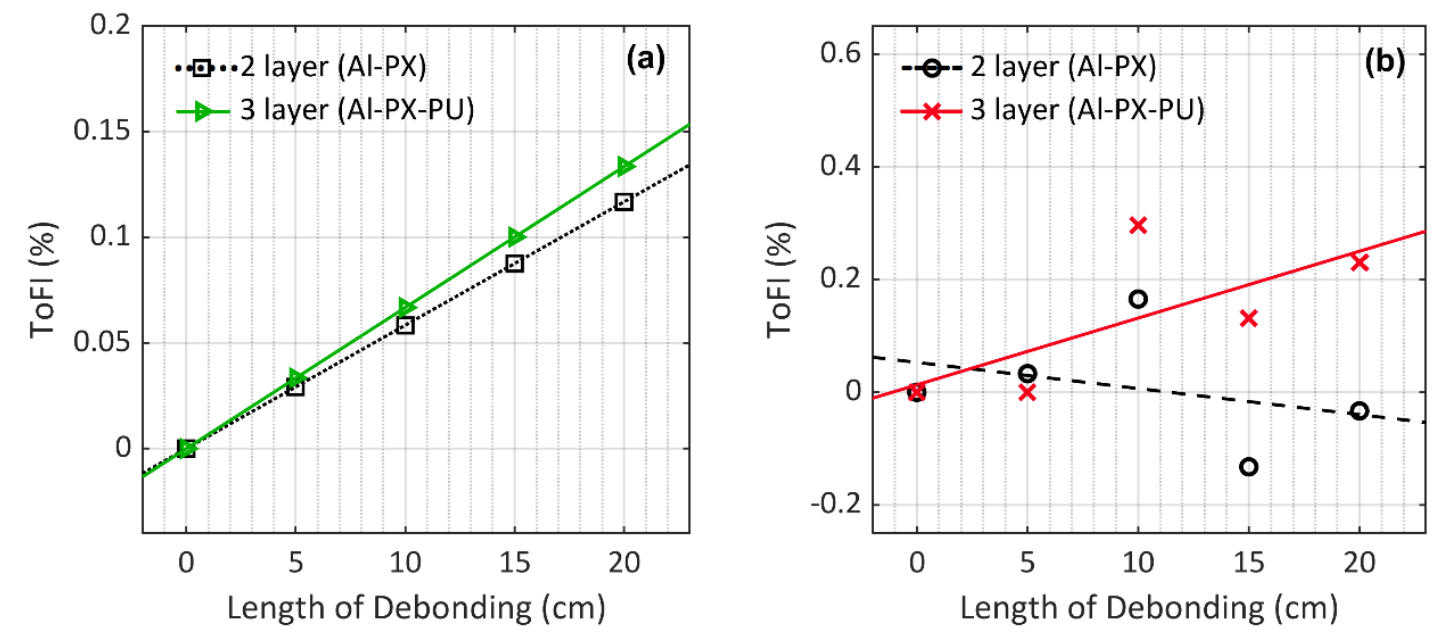

Fig. 9. Time of Flight Index (ToFI) vs. disbond length: (a) theoretical ToFI and (b) experimental ToFI.

\subsection{Sensitivity of Indices in a Noisy Environment}

In order to determine the sensitivity of extraEIcted indices to the disbond defect in a real working situation where the receiving signals might be prone to contamination from noises, additive white Gaussian noise (AWGN) at 20, 10, 0, -10 dB signal-to-noise ratio (SNR) is added. The noise applies to all elements of the full input signal. In a noise-free environment, apart from the ToFI, all indices increase with increasing the length of debonding in both Al-PX and Al-PX-PU cases. In a noisy environment, however, as AWGN distorts the signals, the sensitivity of the indices decreases slightly, especially in two-layer Al-PX that is more affected by the noise. This can be due to the low thickness of the PX adhesive in Al-PX. 

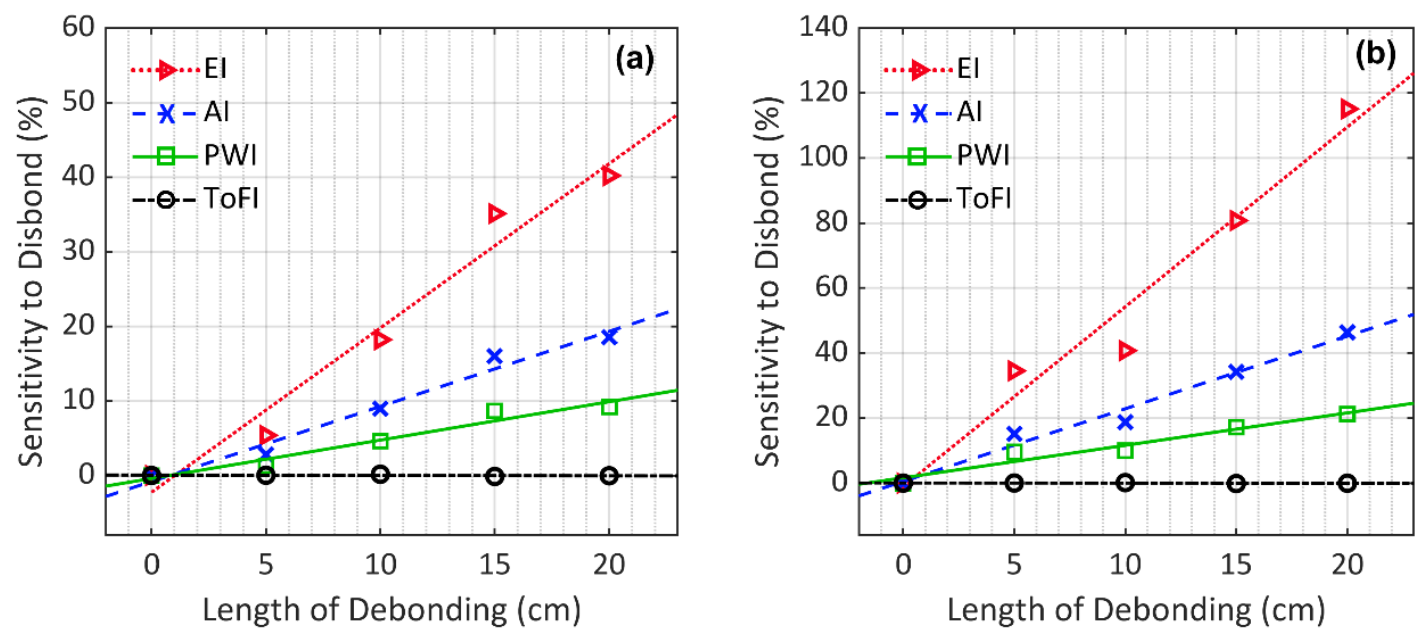

Fig. 10. Comparison of experimental Lamb waves features for disbond detection in a noise-free environment: (a) two-layer Al-PX and (b) three-layer Al-PX-PU.
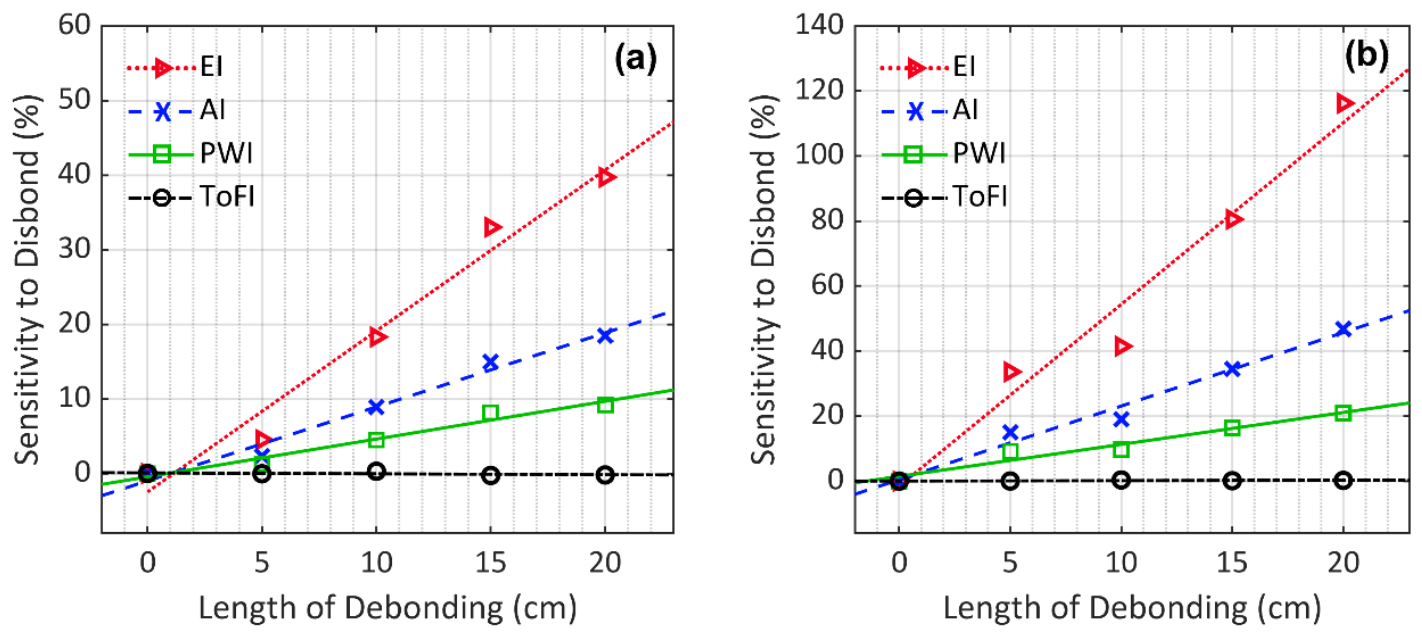

Fig. 11. Comparison of experimental Lamb waves features for disbond detection at $20 \mathrm{~dB}$ SNR: (a) twolayer Al-PX and (b) three-layer Al-PX-PU.
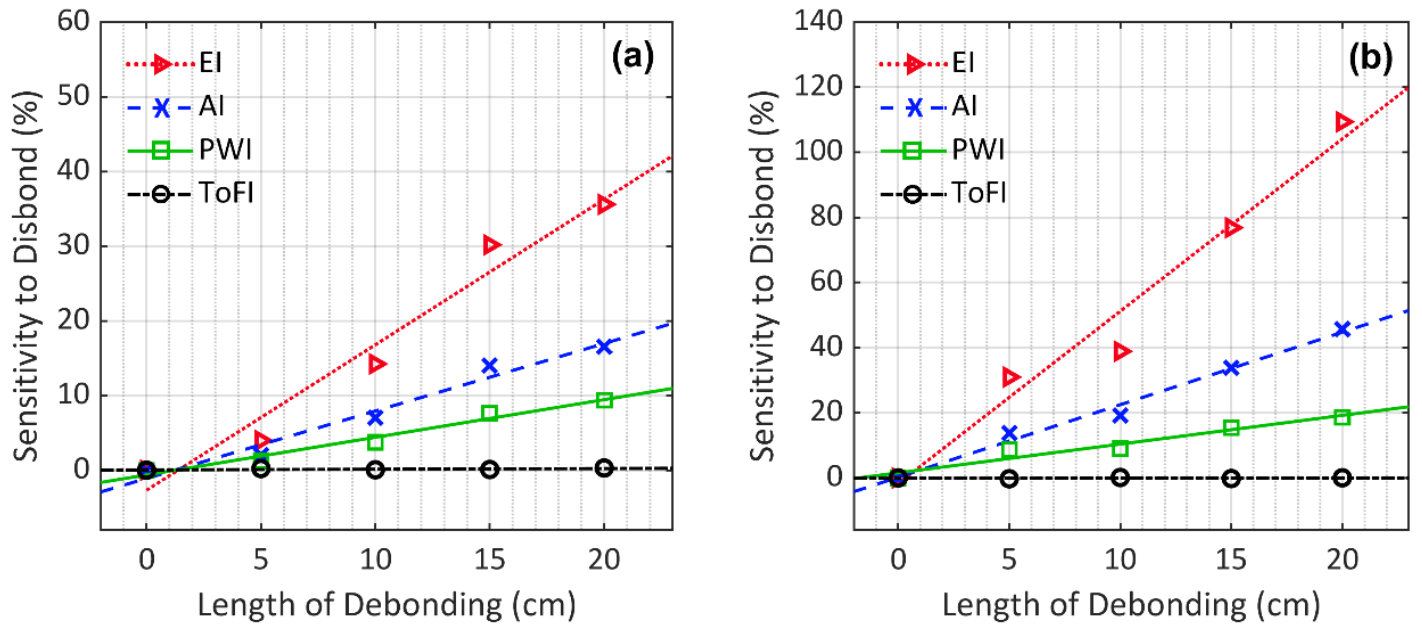

Fig. 12. Comparison of experimental Lamb waves features for disbond detection at $10 \mathrm{~dB}$ SNR: (a) twolayer Al-PX and (b) three-layer Al-PX-PU. 

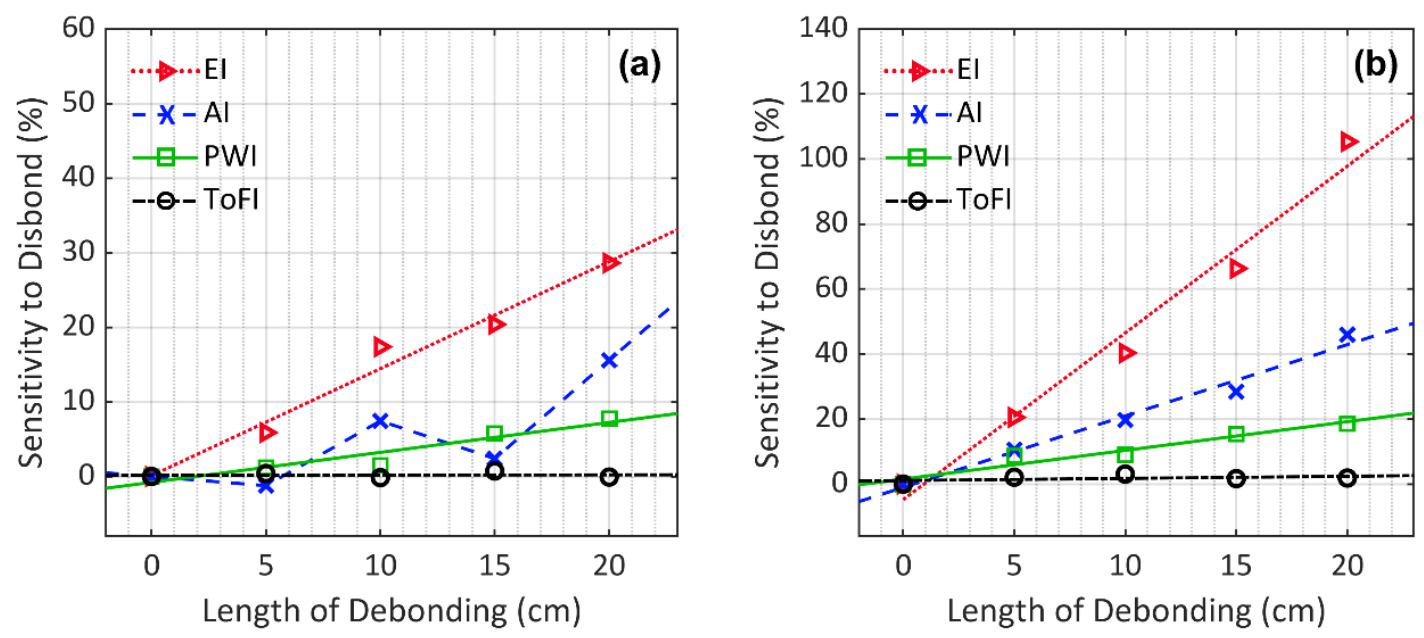

Fig. 13. Comparison of experimental Lamb waves features for disbond detection at 0 dB SNR: (a) twolayer Al-PX and (b) three-layer Al-PX-PU.
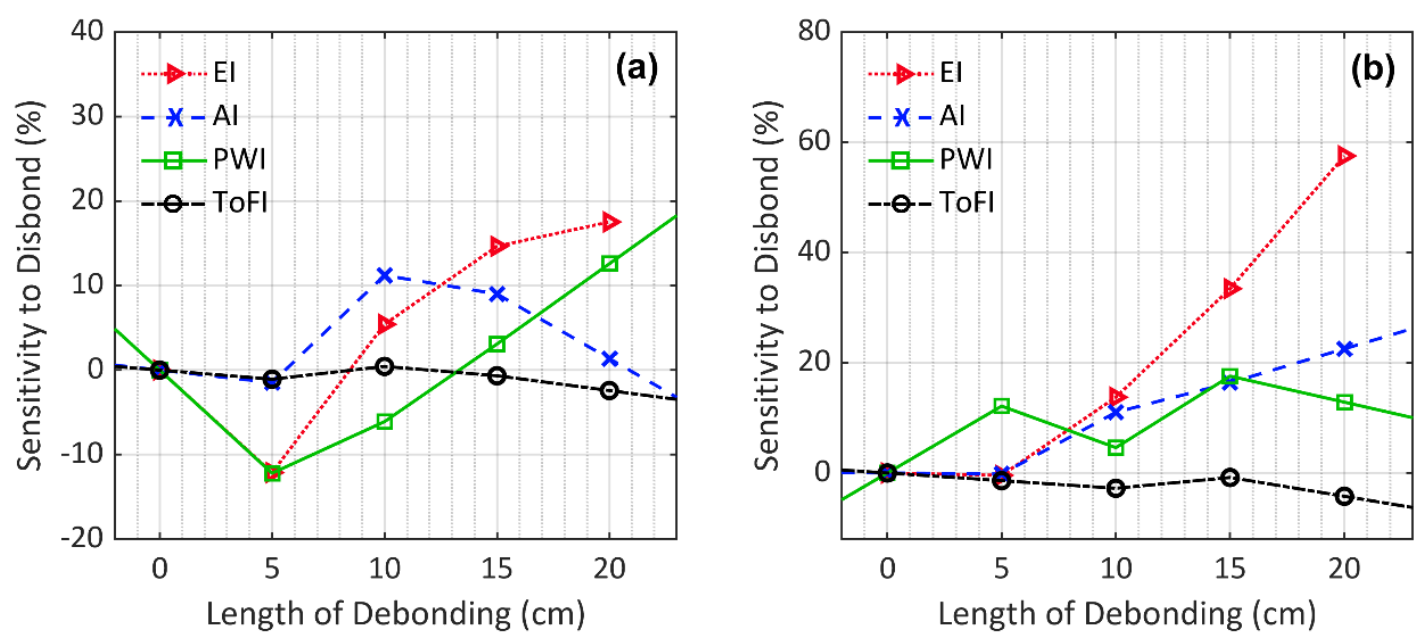

Fig. 14. Comparison of experimental Lamb waves features for disbond detection at -10 dB SNR: (a) twolayer Al-PX and (b) three-layer Al-PX-PU.

Among extracted features, the energy index (EI) is considerably more powerful than the rest, giving in noise-free environment sensitivity rate from 0 to $40 \%$ and from 0 to $115 \%$ in Al-PX and Al-PX-PU, respectively (see Fig. 10). Reducing the efficiency of the EI in case of noisy signal at 20,10, 0 dB SNRs is not noticeable compared to noise-free state (see Fig. 11, Fig. 12, Fig. 13), whereas the EI sensitivity decreases to $18 \%$ and $58 \%$ at -10 dB SNR in Al-PX and Al-PX-PU, respectively (see Fig. 14). As illustrated in Fig. 10 to Fig. 14, except for the 5cm length of debonding at $-10 \mathrm{~dB}$ SNR, the sensitivity of EI to disbond in both the Al-PX and the Al-PX-PU samples in both noise-free and noisy environment is quite robust. Additionally, the EI value is computed by the square of the amplitude that can be also effective in the EI robustness. The interesting fact is that the difference between its value for Al-PX and Al-PX-PU at low-noise state approaches $75 \%$ 
for the most cases, which makes it a valuable feature to distinguish both debonding between a coating and adhesive, and debonding between protective coating (coating and adhesive) and a metal substrate.

The AI is a proper index for debonding evaluation in noisy environment as well but nonetheless its accuracy at 0,-10 dB SNRs in two-layer Al-PX is less than three-layer Al-PX-PU. The efficiency of AI at 20, $10 \mathrm{~dB}$ SNRs are analogous to the noise-free environment, increasing from 0 to $18 \%$ for Al-PX and from 0 to $43 \%$ for AL-PX-PU. There is about $25 \%$ difference between its values for Al-PX and Al-PX-PU cases at lownoise environment, indicating the adhesive and coating contributions in AI individually.

The pulse width index is also able to identify the disbond defect effectively in noisy environment, though not as sensitively as EI and AI. Apart from signals at -10 dB SNR, other noisy states are similar to the noise-free condition, which rises from 0 to $10 \%$ for Al-PX case while it increases from 0 to $20 \%$ for Al-PX-PU.

ToFI is a faint index in comparison with other indices in both noise-free and noisy conditions. Even though the energy of the Lamb waves is absorbed by PU coating, the damping property of this coating affects the arrival time only slightly. In fact, the higher the energy absorption in the viscoelastic material, the longer the arrival time. That is why ToFI is not considered as sensitively as other indices.

The presence of the PU coating has a significant effect on all indices but the striking point is that despite that the PX adhesive is much thinner than the PU coating, its contribution to the wave attenuation is the same as the PU coating in both noise-free and noisy environments. By comparing the Al-PX and Al-PX-PU in Fig. 10 to Fig. 13, it can be seen that this contribution is quite evident in nearly all features. It can be thus inferred that the attenuation of the Lamb waves in the three-layer waveguide (Al-PX-PU) is not simply due to the presence of PU coating, rather the presence of PX adhesive also plays a notable role.

\section{Conclusions}

In this study, ultrasonic Lamb wave was employed experimentally in a two-layer aluminium-adhesive (Al-PX) to examine the ability of Lamb waves modes for detection of debonding defects in a noisy environment. In addition, in order to identify the adhesive contribution in detecting disbonds, the experimental tests have once again performed on 
a three-layer aluminium-adhesive-coating (Al-PX-PU). Using piezoelectric discs, Lamb waves were transmitted and received through the aluminium plate. The SAFE technique was utilized for analysing the mode and frequency, which revealed that at frequencies below $500 \mathrm{kHz}$, the elastic and viscoelastic models of coating have the same behaviour at the first Lamb wave mode (mode 1). It also turned out that the frequency of $350 \mathrm{kHz}$ at mode 1 is the best one in both excitability for the long propagation distance and sensitivity to the disbond defect. Subsequently, a range of important features corresponding to the disbond length were extracted, among which the energy index (EI) was the most robust one, giving sensitivity rates $115 \%, 110 \%$, and $105 \%$ for Al-PX-PU, and $40 \%, 35 \%$, and $30 \%$ for Al-PX in the noisy environment of 20, 10, and $0 \mathrm{~dB}$ SNRs, respectively. The results also shown that the time of flight index (ToFI) was the weakest one. Furthermore, comparing the results in the two-layer waveguide (Al-PX) and threelayer waveguide (Al-PX-PU) proved that the attenuation of the three-layer waveguide (Al-PX-PU) is not merely due to the PU coating, rather the PX adhesive has a remarkable damping capability, giving absorbing rate 30\%, 40\%, and 50\% in EI, AI, and PWI, respectively.

The set of features confirmed that ultrasonic Lamb waves are effective tools for debonding detection between each layer of aluminium, adhesive, and coating, improving the performance of monitoring in noisy environments. This work presents a novel application of Lamb waves along with detailed demonstration on their behaviour when travelling in adhesively bonded structures coated with a viscoelastic layer. The technique is effectively applicable to the inspection of these structures, particularly where adhesives and primers are applied as an intermediate material, such as in pipelines.

Eliminating both PX and PU in the experimental tests was considered as disbond defects. However, using a configuration similar to the one shown in Fig. 1(c), preferably without eliminating both PX and PU in the debonding area, but only PX, helps better identifying debonding failures that can occur in real-world structures. Another direction for further analysis is to explore the sensitivity of frequency-based features.

\section{Acknowledgment}

The authors would like to acknowledge Bocchini and Marzani for their highly useful software GUIGUW [56] to extract the dispersion curves. 


\section{References}

1. Grainger S, Blunt J. Engineering coatings: design and application. Elsevier; 1998.

2. Kinloch AJ. Adhesion and adhesives: science and technology. Springer Science \& Business Media; 2012.

3. Marques E, da Silva LF. Joint strength optimization of adhesively bonded patches. The Journal of Adhesion. 2008;84(11):915-934.

4. Schliekelmann R. Non-destructive testing of adhesive bonded metal-to-metal joints 2. Non-destructive Testing. 1972;5(3):144-153.

5. Rokhlin S, Marom D. Study of adhesive bonds using low-frequency obliquely incident ultrasonic waves. The Journal of the Acoustical Society of America. 1986;80(2):585-590.

6. Guyott C, Cawley P. Evaluation of the cohesive properties of adhesive joints using ultrasonic spectroscopy. NDT International. 1988;21(4):233-240.

7. Pilarski A, Rose JL. A transverse-wave ultrasonic oblique-incidence technique for interfacial weakness detection in adhesive bonds. Journal of Applied Physics. 1988;63(2):300-307.

8. Schindel D. Air-coupled ultrasonic measurements of adhesively bonded multilayer structures. Ultrasonics. 1999;37(3):185-200.

9. Yan D, Drinkwater BW, Neild SA. Measurement of the ultrasonic nonlinearity of kissing bonds in adhesive joints. Ndt \& E International. 2009;42(5):459-466.

10. Tserpes K, Pantelakis S, Kappatos V. The effect of imperfect bonding on the pullout behavior of non-crimp fabric Pi-shaped joints. Computational materials science. 2011;50(4):1372-1380.

11. Steinbild PJ, Höhne R, Füßel R, et al. A sensor detecting kissing bonds in adhesively bonded joints using electric time domain reflectometry. NDT \& E International. 2019;102:114-119.

12. Park B, An Y-K, Sohn H. Visualization of hidden delamination and debonding in composites through noncontact laser ultrasonic scanning. Composites science and technology. 2014;100:10-18.

13. Cawley P, Pialucha T, editors. The prediction and measurement of the ultrasonic reflection coefficient from interlayers in adhesive joints. 1993 Proceedings IEEE Ultrasonics Symposium; 1993: IEEE.

14. Brotherhood C, Drinkwater B, Dixon S. The detectability of kissing bonds in adhesive joints using ultrasonic techniques. Ultrasonics. 2003;41(7):521-529.

15. Adams R, Drinkwater B. Nondestructive testing of adhesively-bonded joints. NDT \& E International. 1997;30(2):93-98.

16. De Angelis G, Dati E, Bernabei M, et al., editors. Development on aerospace composite structures investigation using thermography and shearography in comparison to traditional NDT methods. 2015 IEEE Metrology for Aerospace (MetroAeroSpace); 2015: IEEE. 
17. Su Z, Ye L, Lu Y. Guided Lamb waves for identification of damage in composite structures: A review. Journal of sound and vibration. 2006;295(3-5):753-780.

18. Rose JL. Ultrasonic guided waves in solid media. Cambridge University Press; 2014.

19. Giurgiutiu V. Structural health monitoring of aerospace composites. Academic Press; 2015.

20. Quaegebeur N, Micheau P, Masson P, et al. Structural health monitoring strategy for detection of interlaminar delamination in composite plates. Smart materials and structures. 2010;19(8):085005.

21. Hinders M, Bingham J, editors. Lamb wave pipe coating disbond detection using the dynamic wavelet fingerprinting technique. AIP Conference Proceedings; 2010: AIP.

22. Puthillath P, Rose JL. Ultrasonic guided wave inspection of a titanium repair patch bonded to an aluminum aircraft skin. International Journal of Adhesion and Adhesives. 2010 2010/10;30(7):566-573.

23. Ren B, Lissenden CJ. Ultrasonic guided wave inspection of adhesive bonds between composite laminates. International Journal of Adhesion and Adhesives. 2013;45:59-68.

24. Philtron J, Rose J. Mode perturbation method for optimal guided wave mode and frequency selection. Ultrasonics. 2014;54(7):1817-1824.

25. Giurgiutiu V, Harries K, Petrou M, et al. Disbond detection with piezoelectric wafer active sensors in RC structures strengthened with FRP composite overlays. Earthquake Engineering and Engineering Vibration. 2003;2(2):213-223.

26. Park G, Farrar CR, di Scalea FL, et al. Performance assessment and validation of piezoelectric active-sensors in structural health monitoring. Smart Materials and Structures. 2006;15(6):1673.

27. Rokhlin S, Hefets M, Rosen M. An ultrasonic interface-wave method for predicting the strength of adhesive bonds. Journal of applied physics. 1981;52(4):2847-2851.

28. Lowe M, Challis $\mathrm{R}$, Chan $\mathrm{C}$. The transmission of Lamb waves across adhesively bonded lap joints. The Journal of the Acoustical Society of America. 2000;107(3):1333-1345.

29. Lanza di Scalea F, Rizzo P, Marzani A. Propagation of ultrasonic guided waves in lap-shear adhesive joints: Case of incident a 0 Lamb wave. The Journal of the Acoustical Society of America. 2004;115(1):146-156.

30. Matt H, Bartoli I, Lanza di Scalea F. Ultrasonic guided wave monitoring of composite wing skin-to-spar bonded joints in aerospace structures. The Journal of the Acoustical Society of America. 2005;118(4):2240-2252.

31. Ismaili NA, da Silva CDM, El-Kettani ME-C, et al. Lamb Modes for the Characterization of Bonded Structures. Acta Acustica united with Acustica. 2013 //;99(2):331-334.

32. Spytek J, Ziaja-Sujdak A, Dziedziech K, et al. Evaluation of disbonds at various interfaces of adhesively bonded aluminum plates using all-optical excitation and 
detection of zero-group velocity Lamb waves. NDT \& E International. 2020:102249.

33. Wojtczak E, Rucka M, Knak M. Detection and Imaging of Debonding in Adhesive Joints of Concrete Beams Strengthened with Steel Plates Using Guided Waves and Weighted Root Mean Square. Materials. 2020;13(9):2167.

34. Fromme P, Reymondin J-P, Masserey B. High frequency guided waves for disbond detection in multi-layered structures. Acta Acustica united with Acustica. 2017;103(6):932-940.

35. Zhang K, Zhou Z. Quantitative characterization of disbonds in multilayered bonded composites using laser ultrasonic guided waves. NDT \& E International. 2018;97:42-50.

36. Wang K, Liu M, Cao W, et al. Detection and sizing of disbond in multilayer bonded structure using modally selective guided wave. Structural Health Monitoring. 2019:1475921719866274.

37. Dickstein P, Girshovich S, Sternberg Y, et al. Ultrasonic feature-based classification of the interfacial condition in composite adhesive joints. Research in Nondestructive Evaluation. 1990;2(4):207-224.

38. Nestleroth J, Alers G. Enhanced Implementation of MFL Using EMAT Sensors to Detect External Coating Disbondment. PRCI Contract PR-3-9715 Final Report. 2002:71.

39. Beuker T, Damaschke J. In-line inspection with high resolution emat technology crack detection and coating disbondment. Rosen Technology \& Research Center. 2008;20.

40. Liu M, Chen S, Wong ZZ, et al. In situ Disbond Detection in Adhesive Bonded Multi-layer Metallic Joint Using Time-of-Flight Variation of Guided Wave. Ultrasonics. 2020:106062.

41. Li J, Lu Y, Lee YF. Debonding detection in CFRP-reinforced steel structures using anti-symmetrical guided waves. Composite Structures. 2020;253:112813.

42. Van Velsor JK, Rose JL, Nestleroth JB, editors. Detection of Protective Coating Disbonds in Pipe Using Circumferential Guided Waves. 17th World Conference on Non-destructive testing; 2008: Citeseer.

43. Bostron JH, Rose JL, Moose CA. Ultrasonic guided interface waves at a soft-stiff boundary. The Journal of the Acoustical Society of America. 2013;134(6):4351 4359.

44. Rokhlin S. Lamb wave interaction with lap-shear adhesive joints: Theory and experiment. The Journal of the Acoustical society of America. 1991;89(6):27582765.

45. Ren B, Lissenden CJ. Modal content-based damage indicators for disbonds in adhesively bonded composite structures. Structural Health Monitoring. 2016;15(5):491-504.

46. Bahadori A. Essentials of Coating, Painting, and Lining for the Oil, Gas and Petrochemical Industries. Gulf Professional Publishing; 2015.

47. Jones J. Wave propagation in a two-layered medium. Journal of Applied Mechanics. 1964;31(2):213-222. 
48. Laperre J, Thys W. Experimental and theoretical study of Lamb wave dispersion in aluminum/polymer bilayers. The Journal of the Acoustical Society of America. 1993;94(1):268-278.

49. Simonetti F. Lamb wave propagation in elastic plates coated with viscoelastic materials. The Journal of the Acoustical Society of America. 2004;115(5):20412053.

50. Knopoff L. A matrix method for elastic wave problems. Bulletin of the Seismological Society of America. 1964;54(1):431-438.

51. Lowe MJ. Matrix techniques for modeling ultrasonic waves in multilayered media. IEEE transactions on ultrasonics, ferroelectrics, and frequency control. 1995;42(4):525-542.

52. Barshinger JN, Rose JL. Guided wave propagation in an elastic hollow cylinder coated with a viscoelastic material. ieee transactions on ultrasonics, ferroelectrics, and frequency control. 2004;51(11):1547-1556.

53. Predoi MV, Castaings M, Hosten B, et al. Wave propagation along transversely periodic structures. The Journal of the Acoustical Society of America. 2007;121(4):1935-1944.

54. Bartoli I, Marzani A, di Scalea FL, et al. Modeling wave propagation in damped waveguides of arbitrary cross-section. Journal of Sound and Vibration. 2006;295(3-5):685-707.

55. $\mathrm{Mu}$ J, Rose JL. Guided wave propagation and mode differentiation in hollow cylinders with viscoelastic coatings. The Journal of the Acoustical Society of America. 2008;124(2):866-874.

56. Bocchini P, Marzani A, Viola E. Graphical user interface for guided acoustic waves. Journal of Computing in Civil Engineering. 2010;25(3):202-210.

57. Murayama T. Dynamic mechanical analysis of polymeric material. Elsevier Amsterdam; 1978.

58. Schubert KJ, Herrmann AS. On attenuation and measurement of Lamb waves in viscoelastic composites. Composite Structures. 2011;94(1):177-185. 